\title{
Generalized Lead-Acid based Battery Model used for a Battery Management System
}

\author{
By Philip Dost ${ }^{*}$ \\ Constantinos Sourkounis ${ }^{t}$
}

This paper deals with the determination of a battery model for different designs of lead-acid based batteries. Although batteries with gelled electrolyte and absorbent glass mat (AGM) batteries are based on the chemistry of common leadacid batteries they differ in regards to the parameters of a battery model. In this paper several measurement methods for the identification of these parameters are presented. These parameters enable the establishment of a battery model. A precise battery model can provide the basis for a battery management system (BMS). A BMS is required due to the rising number of electrical components in modern vehicles making it necessary to control the current capability of the battery. The results presented in this paper indicate that every battery type needs a specially optimized battery management system as the behaviour and the characteristic qualities vary between the different designs.

Keywords: Absorbent glass mat, automotive applications, battery management systems, energy storage, valve-regulated lead-acid battery

\section{Introduction}

Batteries which are based on lead-acid technology are generally deployed in any passenger vehicle. These batteries are mandatory for the running of standard combustion engines and therefore are often observed by a so called battery management system (BMS) (Kerley et al., 2015) in modern vehicles, to ensure they remain in good working order (Cugnet et al., 2010). Such a control unit is necessary in order to ensure that the battery can provide enough power for the rising number of electrical components by way of a continuous analysis of several states of the battery.

The different designs such as the classic flooded lead-acid battery (Salkind and Zguris, 2011) as well as valve-regulated lead-acid batteries (Bullock and Salkind, 2011; Berndt, 2001; Lambert et al., 2002; Zhang et al., 2012) have been the subject of a great variety of research. Many approaches have been taken in order to describe the behaviour of batteries and certain effects either by mathematical (Gu, 1990; Salameh et al., 1992; Mariani et al., 2015) or equivalent circuit models (Mauracher and Karden, 1996; Buller et al., 2003; Eifert, 2014).

\footnotetext{
*Scientific Assistant, Ruhr-University Bochum, Germany.

* Head of EneSys Institute, Ruhr-University Bochum, Germany.
} 
Nevertheless most state estimations are based on only one type of lead-acid battery design. Therefore the impact of the design variation on a BMS has not yet been stated in detail. This is an important consideration, because, even though all types of lead-acid based batteries depend on the same chemistry they do not all have the same behaviour.

As a BMS can only be as precise as the underlying battery model, special adaptions and optimizations must be implemented in regards to the battery model for different types of batteries. Therefore the objective of this paper is to present results regarding the challenges which a variation of the battery design implies for an exact matching of the underlying battery model.

The structure of the paper is as follows: The following section discusses the different designs of lead-acid based batteries and their applications. Section "Battery Model" presents a general battery model and the determination of the model's parameters. In section "Description of the Battery Status" different values which are used in order to describe the charge status of the battery are explained. Section "Measurement Methods" deals with the applied measurement methods and their evaluation. Finally, the last section summarizes the results and provides a conclusion.

\section{Lead-Acid Based Batteries}

Commonly used batteries in vehicles are lead-acid batteries. They are widespread and therefore offer a good cost-benefit ratio. Nevertheless they suffer from several disadvantages, such as a lower number of cycles compared to other technologies and hydrogen evolution which can be an explosion hazard (Salkind and Zguris, 2011). In addition, they are not maintenance-free, as water must be refilled and they may not be operated or stored in any other, but the provided position due to the liquid electrolyte (Salkind and Zguris, 2011). A good alternative, in order to overcome some of the disadvantages of this classic type of lead-acid battery are valve-regulated lead-acid batteries (VRLA), such as absorbent glass mat (AGM) and batteries with gelled electrolyte (GEL) (Bullock and Salkind, 2011). This sealed design can be achieved due to an internal oxygen cycle which is able to absorb overcharging current as oxygen cannot escape from the cell. This internal oxygen cycle is achieved with the help of the so called immobilization of the electrolyte. Nevertheless, a totally sealed design is not possible as small portions of gas still do escape due to hydrogen evolution (Berndt, 2001).

This is the reason why these batteries are characterized by a pressure-relief valve which only works one-way and seals the cell, up to the maximum of the internal pressure (Bullock and Salkind, 2011). Therefore, VRLAs are maintenance-free and their operating position can be either vertical or horizontal as there is no liquid electrolyte within the cell (Bullock and Salkind, 2011; Berndt, 2001). 
Although VRLAs differ from the original flooded lead-acid batteries they are still based on the same chemistry. Accordingly, the chemical reactions are as follows (Bullock and Salkind, 2011).

The reaction at the negative electrode is

$$
\mathrm{Pb}+\mathrm{SO}_{4}^{2-} \underset{\text { Charge }}{\rightleftharpoons} \mathrm{PbSO}_{4}+2 \mathrm{e}^{-}
$$

and at the positive electrode

$$
\mathrm{PbO}_{2}+4 \mathrm{H}^{+}+\mathrm{SO}_{4}^{2-} \underset{\text { Charge }}{\rightleftharpoons} \mathrm{PbSO}_{4}+2 \mathrm{H}_{2} \mathrm{O}
$$

This leads to the overall reaction

$$
\mathrm{Pb}+\mathrm{PbO}_{2}+2 \mathrm{H}_{2} \mathrm{SO}_{4} \underset{\text { Charge }}{\rightleftharpoons} 2 \mathrm{PbSO}_{4}+2 \mathrm{H}_{2} \mathrm{O}
$$

As previously mentioned, the difference between the classic lead-acid battery and the VRLA battery is that most of the oxygen which is generated within the cells at normal overcharge rates is recombined inside the cell. For a more detailed description of the chemical processes see (Bullock and Salkind, 2011; Berndt, 2001).

In the following two sections the different ways of immobilizing the electrolyte as well as the advantages and disadvantages of AGM and batteries with gelled electrolyte are presented.

\section{Absorbent Glass Mat}

In AGM batteries the immobilization of the electrolyte is achieved by absorption in highly porous AGM separators between the electrodes. These separators are made from glass microfibers through which the generated oxygen can pass. This reduces hydrogen evolution as it allows the oxygen to move from the positive to the negative electrode where it reacts to water (Bullock and Salkind, 2011).

AGM batteries provide a smaller internal resistance than the flooded design (Lambert et al., 2002) and therefore high cold cranking amps (CCA). That is the reason why they are often used in vehicles with a start-stop function.

\section{Gelled Electrolyte}

In batteries with gelled electrolyte, the electrolyte is immobilized by using silicic acid $\left(\mathrm{SiO}_{2}\right)$ as an additive (Berndt, 2001). This mixture is poured into the cells, where it hardens. A network of cracks which is caused by dry-out makes the transition of oxygen between the electrodes possible (Bullock and Salkind, 2011). This design implies a higher internal resistance in comparison to the other lead based batteries (Zhang et al., 2012). That is why the CCA is very limited and this battery is only usable with restrictions as a starter battery in a vehicle. 


\section{Battery Model}

As mentioned in the previous section, a good knowledge of the behaviour of the battery is necessary for a reliable performance of the BMS.

The battery as a chemical storage system features a chemically influenced and non-linear behaviour. This behavior is affected by different conditions such as the state of charge (see subsection "State of Charge"), the method of charging as well as the attached load. In order to describe these properties, the battery can be modeled with the help of an equivalent circuit diagram consisting of different electric circuit elements. As a frequency analysis is quite difficult to realize with batteries, an impedance spectroscopy (Mauracher and Karden, 1996) can be regarded as a good alternative by providing a Nyquist diagram which allows the determination of the elements of an equivalent circuit diagram.

Figure 1. Generalized Lead-Acid Batteries Nyquist Diagram Resulting from and Impedance Spectroscopy

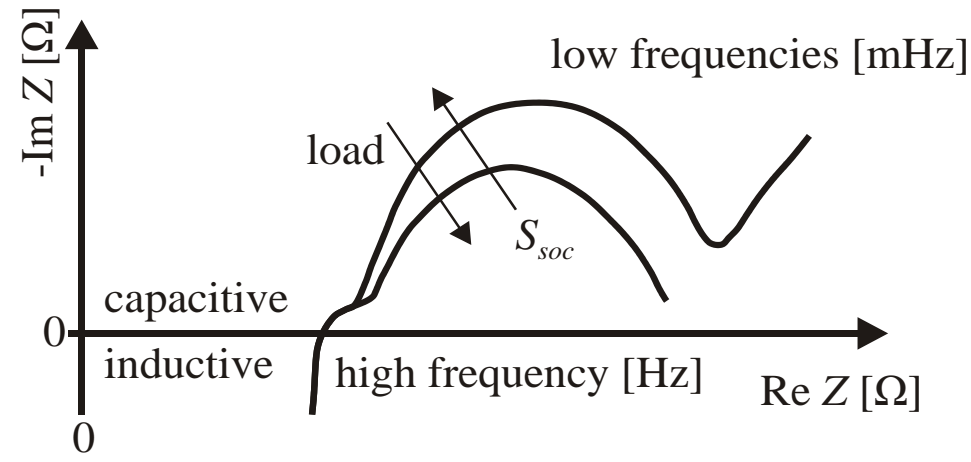

With the help of the impedance spectroscopy, an impedance graph can be acquired. A qualitative course of such a graph is presented in Figure 1. It shows an inductive behaviour for high frequencies and a capacitive behaviour for lower frequencies. Accordingly, a simplified battery model can be realized with the internal resistance $R_{i}$ to model the conductive media of the battery (Mauracher and Karden, 1996), an inductive impedance $L$ due to the metallic connection between the electrodes and the poles (Buller et al., 2003) and a parallel RC-circuit (see Figure 2). The resistor $R_{d}$ stands for the charge-transfer resistance whereas the capacitor $C_{d}$ models the double-layer capacitance of the electrolyte.

In addition a Zarc element $Z_{k i}$ must be applied to the representing circuit due to the capacitive behaviour and semicircle in the complex-plane. This element represents an infinite number of circuit elements. However, a limited amount of RC-elements does allow a good approximation of the batteries behaviour.

As a battery is an energy source, the essence of this model is the voltage source $U_{0}$ which is a function of the state of charge and represents the open circuit voltage. Due to the load on the battery and its chemical behaviour this open circuit is overlaid by overvoltages which can be ascribed to the elements of the equivalent circuit diagram. In combination, these voltages build the terminal voltage $U_{\text {bat }}$. 
Figure 2. Equivalent Circuit Diagram

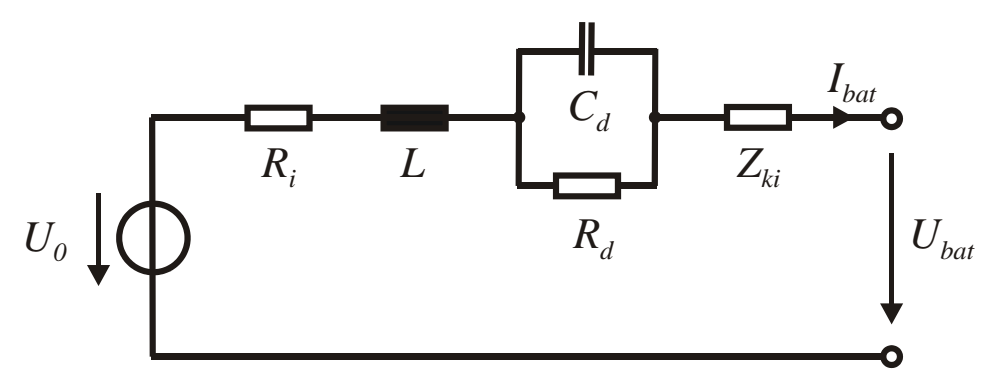

Due to the various time constants of the elements, the open circuit voltage denotes the voltage, being measured, without any load and after all processes have been completely decayed.

The presented battery model can be regarded as valid for all known types of lead-acid based batteries, however the parameters of the introduced elements change and as a consequence must be determined for the different types. For this purpose the measurement methods which are required for such a determination of the parameters are presented in section "Measurement Methods".

\section{Description of the Battery Status}

\section{State of Charge}

The state of charge $S_{s o c}$ (S for state) denotes the amount of energy which is stored in the battery, as a percentage or as a ratio. It is a highly important value which must be determined by the BMS as a direct measurement is not possible.

For this purpose the open circuit voltage of the battery is applied. This is a virtual voltage which cannot be measured and therefore must be calculated from the terminal voltage and by subtraction of all occurring overvoltages. These overvoltages can be estimated with the help of the presented battery model.

For the maximum open circuit voltage the $S_{s o c}$ assumes the value 1 or $100 \%$, whereas the minimum allowed open circuit voltage is represented by 0 or $0 \%$.

It is important to mention that there is not necessarily a direct relation between the charge and the voltage, as most of the batteries have a non-linear voltage drop.

$$
\delta Q \neq \delta u_{o c}
$$

Accordingly, the $S_{s o c}$ must be calculated in relation to the charge $(Q)$.

$$
S_{\text {soc }} \sim Q
$$

\section{State of Discharge}

Another significant value is the depth of discharge $S_{\text {dod }}$ which includes the same information as the state of charge. However, it shows the analogue amount of discharge and therefore varies between 0 or $0 \%$ for a fully charged battery 
$\left(\mathrm{S}_{\mathrm{soc}}=100 \%\right)$ and 1 or $100 \%\left(\mathrm{~S}_{\mathrm{soc}}=0 \%\right)$ for an empty battery respectively. Accordingly, the relation is given by

$$
S_{\text {dod }}=1-S_{\text {soc }}
$$

\section{Measurement Methods}

In order to determine the parameters of the battery model as well as the behaviour of the battery while charging, discharging and being exposed to changing loads, several measurement methods were applied. In this section the test bed and its different setups are presented.

The employed batteries and their characteristics are summarized in Table 1.

Table 1. Employed Lead-Acid Based Batteries

\begin{tabular}{|c|c|c|c|c|}
\hline Manufacturer & Type & $\begin{array}{c}\text { Nominal } \\
\text { Capacity } \\
{[\mathrm{Ah}]}\end{array}$ & $\begin{array}{c}\text { Nominal } \\
\text { Voltage } \\
{[\mathrm{V}]}\end{array}$ & $\begin{array}{c}\text { Cold Crancing } \\
\text { Current [A] }\end{array}$ \\
\hline \hline Arktis & Lead-Acid & 88 & 12 & 640 \\
\hline Banner & $\begin{array}{c}\text { Absorbent Glass Mat } \\
\text { (AGM) }\end{array}$ & 92 & 12 & 850 \\
\hline Exide & Gelled Electrolyte & 80 & 12 & 540 \\
\hline
\end{tabular}

The batteries were analysed with the help of an adjustable test bed. The measurement methods and the obtained results are described in detail in the following part of the paper.

\section{Charging Discharging and Load Measurements}

The test bed has the ability to measure the battery voltage $U_{b a t}$ and battery current $I_{b a t}$ with an adjustable sampling rate of up to $225 \mathrm{kHz}$ while the battery is charging, discharging or exposed to changing loads.

The process of charging is performed with a charging device that applies the i-u-charging mode. This method begins with current controlled charging and switches to voltage controlled charging between a $S_{s o c}$ of $50 \%$ to $80 \%$ to avoid overload (Dost and Sourkounis, 2012). 
Figure 3. Test Bed Setup for Discharging and Load Measurements of a Battery

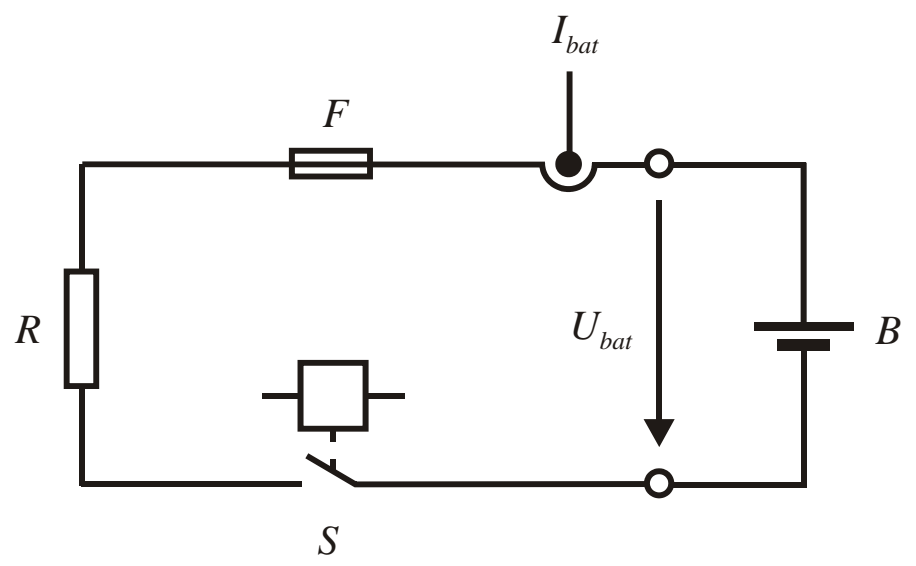

For controlled discharging of batteries, the setup shown in Figure 3 is employed. The battery is preserved against overcurrent by the fuse $F$. It consists of a variable array of resistors represented by the resistor $R$ which is connected to the battery with the help of an electric contactor $S$. By modifying the resistance array, different load currents can be set to affect the battery. The variable array of constant resistors as a load causes a voltage dependent current. For performing the measurements this has no significant influence, but offers the advantage to discharge the battery with different currents.

Figure 4 shows the course of the voltage and current while discharging as well as the following charging process after a short stationary phase with no load current. Measuring a discharging process allows the derivation of a linear course of the open circuit voltage. This can be used in order to estimate the related open circuit voltage for every $S_{s o c}$.

In addition, batteries can be discharged with different intervals of interruptions during the discharging phase when using the resistor as a load. Thereby, an automated discharge of a battery in variable step sizes regarding the capacity is performed. An appropriate length of a period of dormant phase after the discharging phase allows the overvoltages to decay and a determination of the open circuit voltage for each of the steps with relation to the $S_{s o c}$ is possible. 
Figure 4. Course of Voltage and Current of an AGM Battery while Discharging and Following Charging

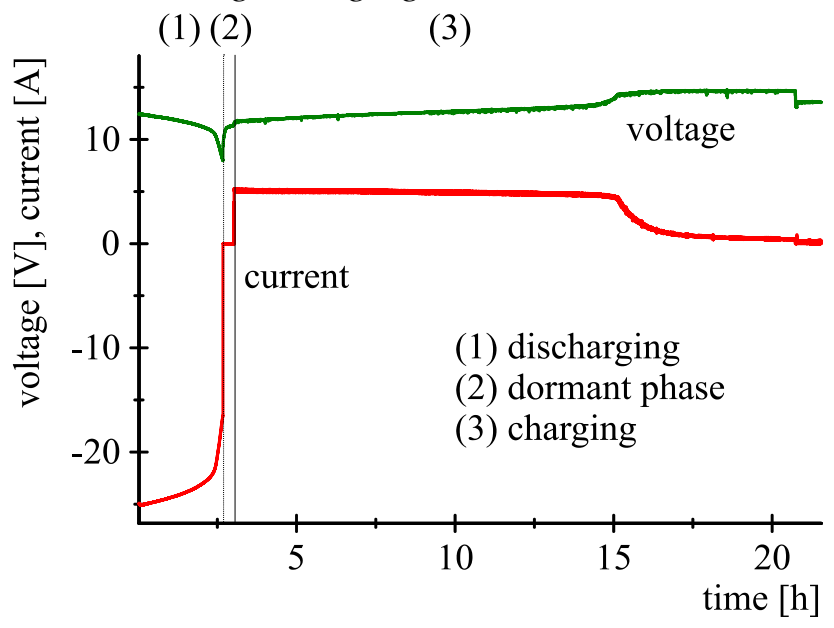

Figure 5 shows a discharging process with discharging intervals of 45 minutes with a current of approximately $5 \mathrm{~A}$ and stationary phases of about 2 hours. A safety mechanism terminates the discharging measurement when the end-of-discharge voltage is reached and further withdrawal of charge would damage the battery.

The values of the open circuit voltage have been extracted from the measurement and plotted as a function of the $S_{\text {soc }}$ in Fig. 6 . The course of the open circuit voltage is linear for a wide range of the measurement except for a sharp drop towards lower values of the $S_{s o c}$. The presented course of the analysed AGM battery may not be regarded as generally valid, as all batteries differ concerning the correlation between open circuit voltage and $S_{s o c}$.

Figure 5. Course of Voltage and Current of an AGM Battery while Discharging in Intervals

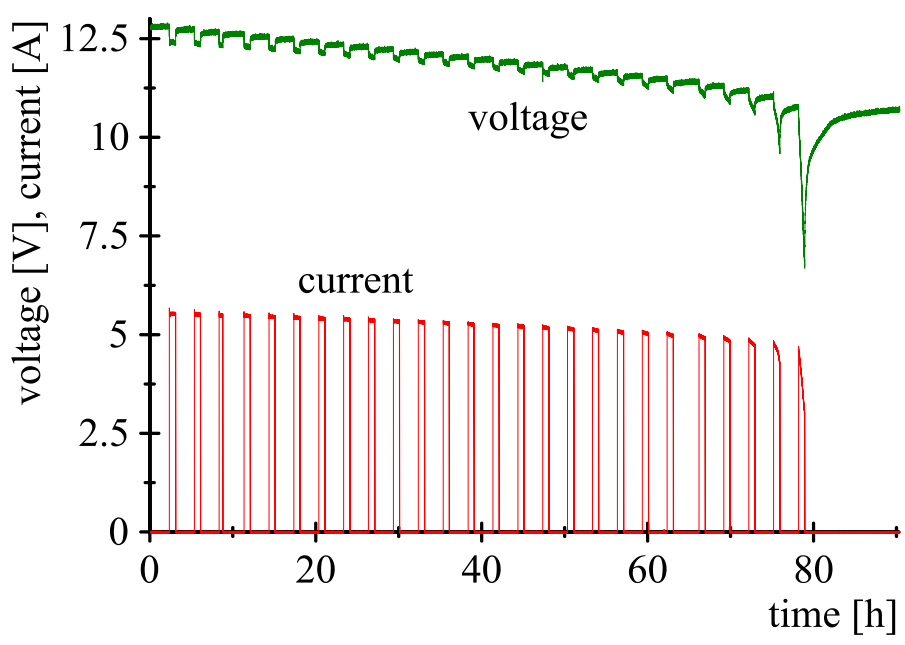


A good knowledge of this relation is essential for a BMS, as it promises good results for calculating the remaining charge of the battery in combination with other methods such as the coulomb counting method (Piller et al., 2001).

Figure 6. Course of the Open Circuit Voltage for an AGM Battery as a Function of $S_{\text {soc }}$

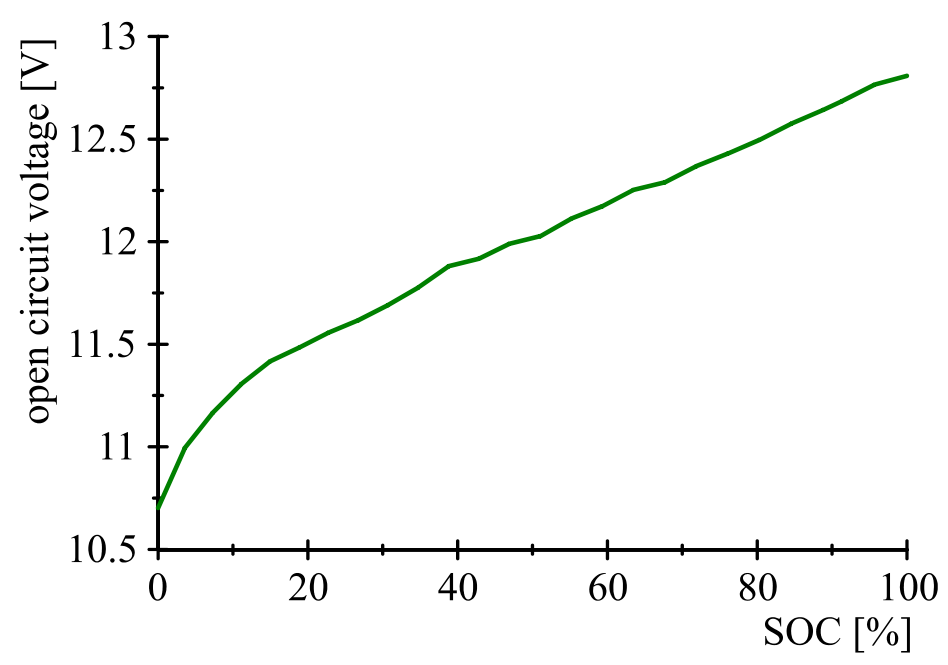

Impedance Spectroscopy

The description of the theory behind the electrochemical impedance spectroscopy (EIS), which is described below, is based on (Salomon, 2011). The galvanostatic method of the EIS employs an AC current in order to determine the impedance $Z$, by measuring the voltage feedback of the battery for a wide frequency range. Accordingly, the impedance is given by

$$
Z(f)=\frac{v_{b a t}(f)}{I_{\text {bat }}(f)}
$$

The complex impedance consists of the real part $Z$ ' and the imaginary part $Z$ '” as the following equation shows

$$
Z(f)=Z^{\prime}(f)+Z^{\prime} \quad(f)(8)
$$

With the help of a fast Fourier transform (FFT) the measured AC signals for current and voltage can be analysed and the result delivers a real part and imaginary part of the impedance $Z$.

These results can be transferred into a Nyquist diagram. This implies that for every measured frequency, the real part of the complex impedance is plotted against the imaginary part of the complex impedance. 
Figure 7. Test Bed Setup for Undertaking an Impedance Spectroscopy of a Battery

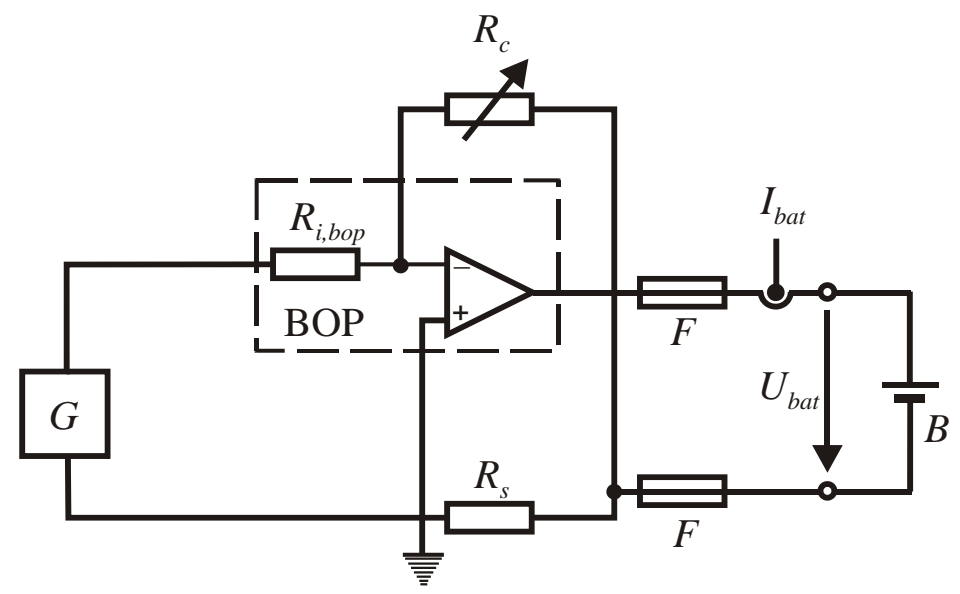

The test bed setup for performing an impedance spectroscopy is illustrated in Figure 7. The frequency generator $G$ delivers an AC voltage signal which is transformed into an AC current signal with the help of the bipolar operational power supply (BOP). $R_{i, b o p}$ describes the internal resistance of the BOP. The wiring of the operational amplifier is assembled from the constant resistor $R_{s}$ and the adjustable sliding resistance $R_{c}$ and therefore allows a variable amplification.

Figure 8. Determination of the Equivalent Circuit Diagram's Elements with the Help of the Nyquist Diagram

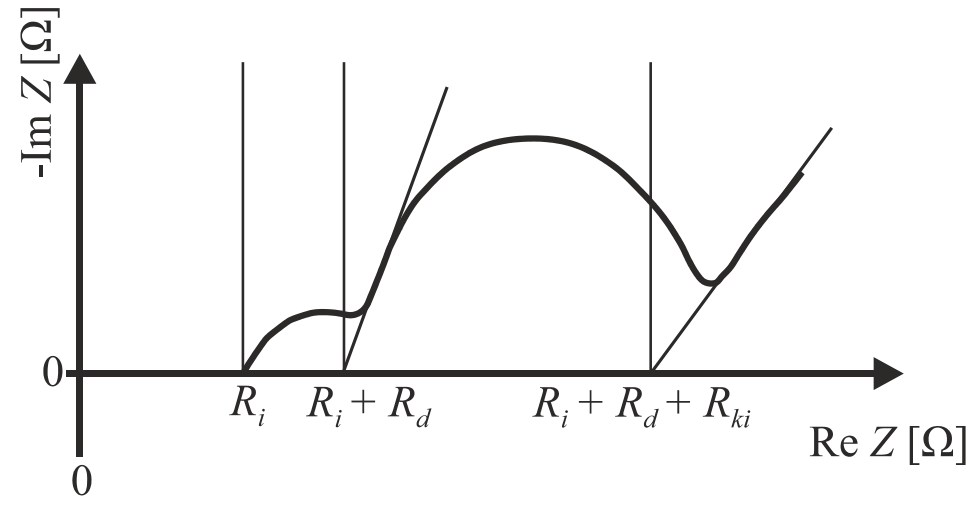

Figure 8 shows how the parameters $R_{i}, R_{d}$ and $R_{k i}$ of the equivalent circuit diagram can be determined in a Nyquist diagram on the basis of a qualitative course.

The internal resistance is identified at the point of intersection of the $\mathrm{x}$-axis and the locus, as the battery shows a pure ohmic behaviour. Tangents are placed at the slopes of the locus in order to determine the values of the elements $R_{d}$ and $R_{k i}$.

As Figure 9 shows, the three types of lead-acid based batteries feature different courses of the locus concerning the position in the coordinate system. 
The course of the AGM battery cuts the axis of abscissas at a smaller value than the other two courses. Therefore the AGM battery has an inferior internal resistance. The GEL battery has the highest internal resistance.

Furthermore, a determination of the parameters - as presented in Figure 8 leads to different values of the other elements for the various battery types. The equivalent circuit diagram remains the same, however the behaviour of the battery changes.

Figure 9. Nyquist Diagram of an AGM, a GEL and a Lead-Acid Battery for a Frequency Range from $50 \mu \mathrm{Hz}$ to $12.5 \mathrm{kHz}$ (top) and an Excerpt, Illustrating the Intersect of the Graphs and the Abscissa in Detail (Bottom)

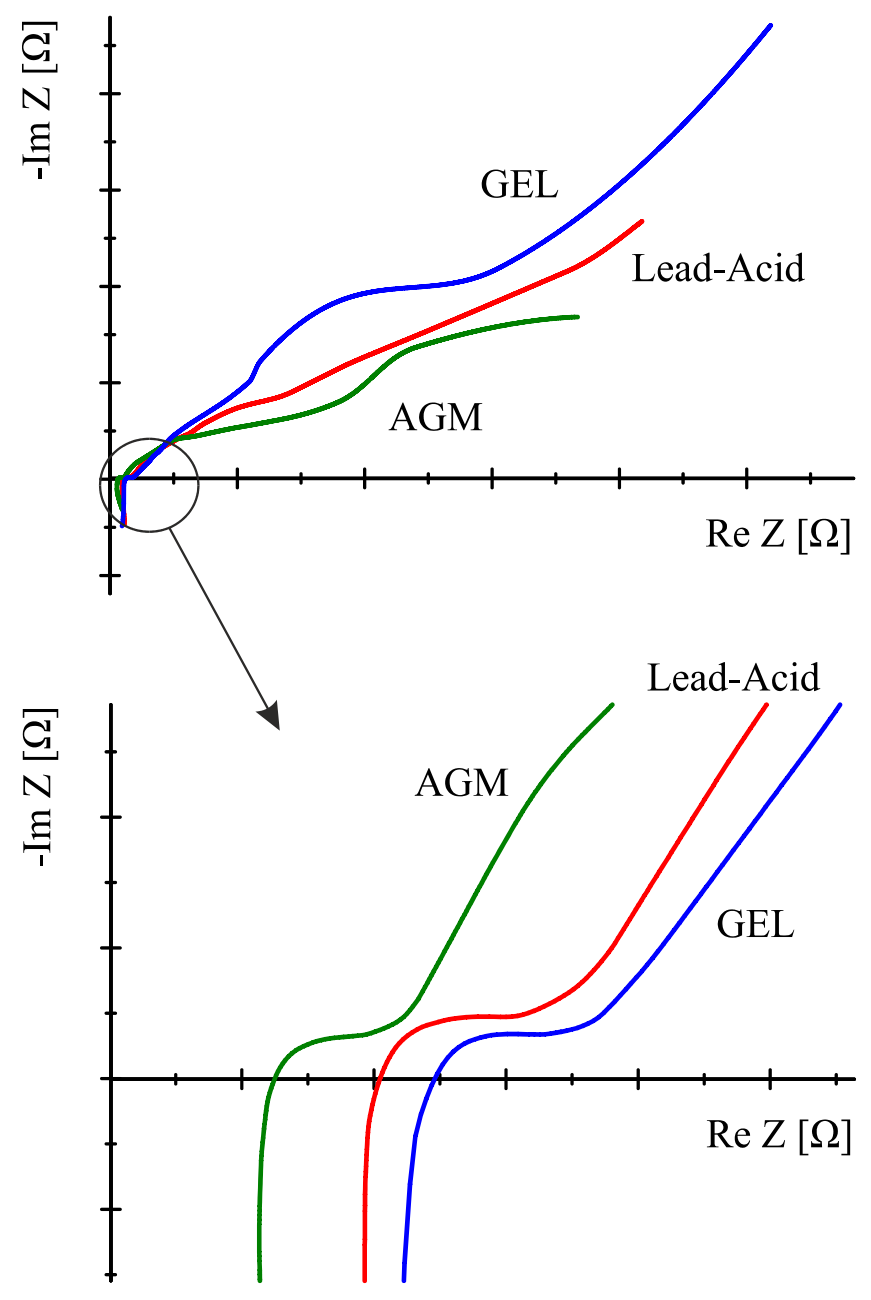

An additional effect that can be shown with the help of the Nyquist diagram in Figure 10 is the dependence on the $S_{s o c}$. The upper part of the diagram reveals the results of an impedance spectroscopy of an AGM battery at different values of $S_{s o c}$. Noticeably the locus of a completely charged battery $\left(S_{s o c}=100 \%\right)$ departs very clearly from the other states of charge. This is important in regards to a 
determination of the parameters when the elements are regarded as independent of the state of charge, for reasons of simplification. In this case the $S_{s o c}$ of $100 \%$ should be disregarded.

The lower illustration of Figure 10 shows that the graphs of different $S_{s o c}$ values cut the axis of abscissas at different points. This means that the internal resistance of the battery depends on the state of charge and increases when the remaining capacity decreases. This effect must be considered when the internal resistance is used by the BMS in order to calculate the overvoltage for the element $R_{i}$ of the equivalent circuit diagram. Furthermore, the internal resistance can be used to determine the state of health of a battery (Iwata, 1997; Ushiyama and Morimoto, 2011) and therefore must be observed continuously while taking the boundary conditions, such as temperature or state of charge into account.

Figure 10. Nyquist Diagram of an AGM Battery for the Frequency Range of 2.5 $m \mathrm{~Hz}$ to $12.5 \mathrm{kHz}$ at Different Values of $S_{\text {soc }}($ Top) and an Excerpt (Bottom)

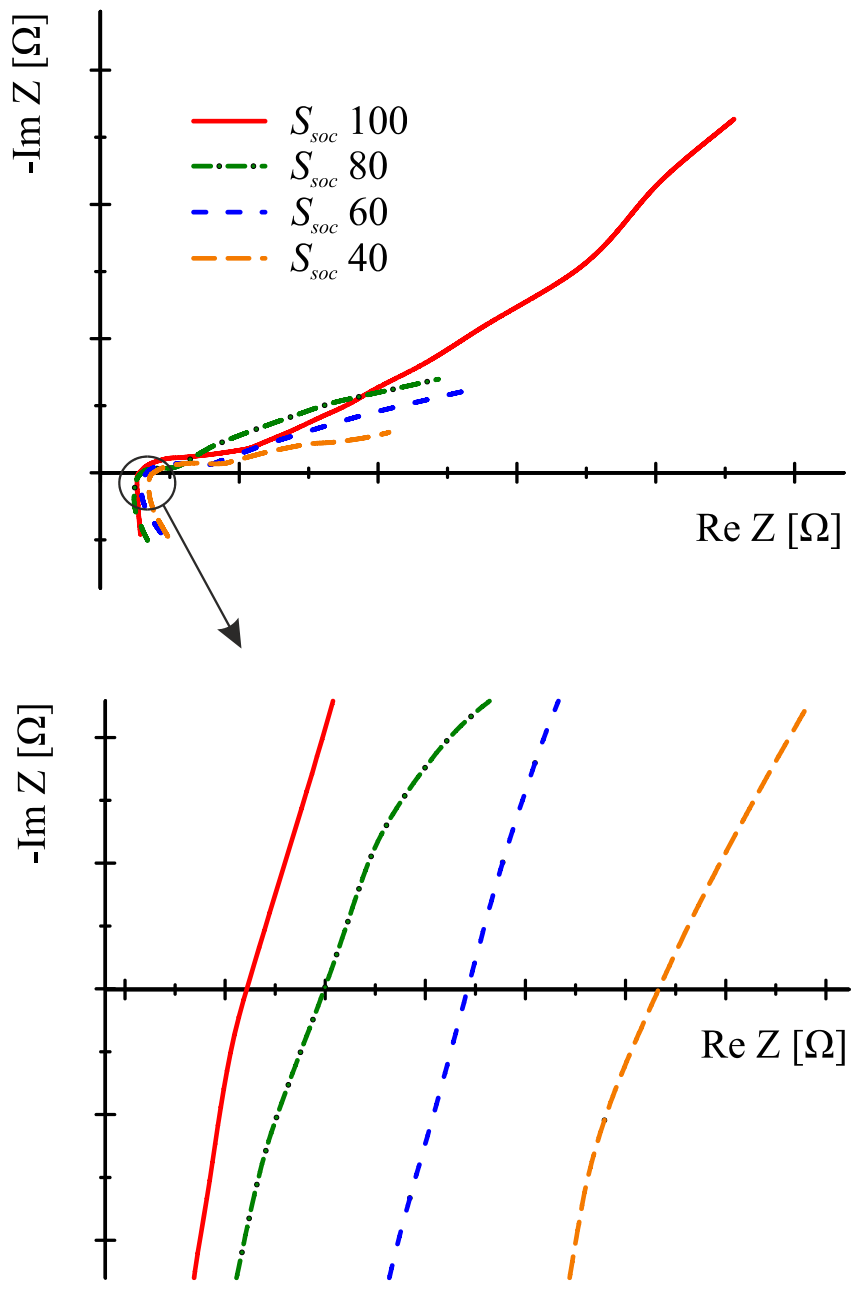

Table 2 summarizes the results of the impedance spectroscopy. The low internal resistance of the AGM battery corresponds with the high value of the cold 
cranking current. Likewise, the cold cranking current is smaller with a gelled electrolyte as present in the Exide battery. Towards higher frequencies the internal resistance in the presented AGM-Battery rises more moderately than in the presented gelled battery, while the capacitive behaviour remains below the gelled battery at high frequencies.

Table 2. Summarized Measurement Results of the Employed Lead-Acid Based Batteries

\begin{tabular}{|c|c|c|c|c|}
\hline Manufacturer & Type & $\begin{array}{c}\text { Internal } \\
\text { Resistance }\end{array}$ & High frequencies & $\begin{array}{c}\text { Low } \\
\text { frequencies }\end{array}$ \\
\hline \hline Arktis & Lead-Acid & Medium & - & $\begin{array}{c}\text { Medium level } \\
\text { capacitive }\end{array}$ \\
\hline Banner & $\begin{array}{c}\text { Absorbent Glass } \\
\text { Mat (AGM) }\end{array}$ & Low & $\begin{array}{c}\text { Smaller internal } \\
\text { resistance and capacitive } \\
\text { bahaviour }\end{array}$ & $\begin{array}{c}\text { Least } \\
\text { capacitive }\end{array}$ \\
\hline Exide & $\begin{array}{c}\text { Gelled } \\
\text { Electrolyte }\end{array}$ & high & $\begin{array}{c}\text { Higher internal resistance } \\
\text { and capacitive behaviour }\end{array}$ & $\begin{array}{c}\text { Most } \\
\text { capacitive }\end{array}$ \\
\hline
\end{tabular}

Figure 11 shows a validation of the state of charge estimation of the BMS. Therefore, the AGM battery has been discharged stepwise, down to the end-ofdischarge voltage. The $S_{\text {soc,real }}$ has therefore been extracted from the measured values according to the real discharged and charged capacity. The $S_{\text {soc,BMS }}$ shows the estimated $S_{\text {soc }}$ of the BMS. Despite a minor error of maximal $1 \%$ the $S_{\text {Soc,BMS }}$ automatically adjusts frequently at a fully charged and discharged battery. This guarantees a continuously good prediction of the $S_{\text {soc }}$.

Figure 11. $S_{s o c}$ Validation of a BMS with Open Voltage Estimation of the AGM Battery

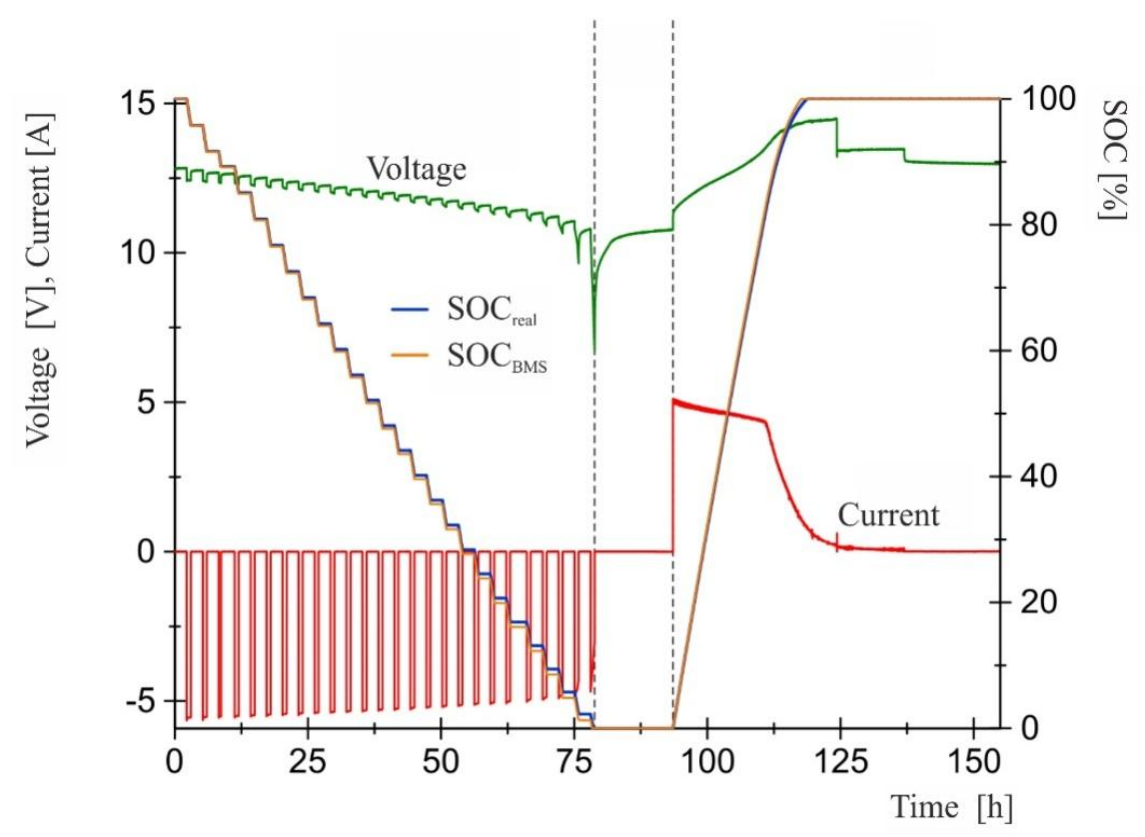




\section{Conclusions}

In this paper a battery model for all types of lead-acid based batteries is considered. Therefore an equivalent circuit diagram, as well as different values which are used in order to describe the status of the battery, have been introduced.

It has been shown that the parameters of this model change when another type of battery, for example an AGM or GEL battery instead of a classic lead-acid battery, is employed. A test bed with variable setup options for different measurement methods which allows measuring of characteristic curves has been presented. Furthermore, a way of determining the parameters with the help of the acquired results has been shown.

The link between the state of charge and the open circuit voltage has been examined by performing discharging measurements. With the help of the open circuit voltage it is possible to determine the remaining capacity of a battery, which cannot be measured directly. As a further effect, the influence of the state of charge on the internal resistance has been exposed by the results of an electrochemical impedance spectroscopy. Understanding the behaviour of various types of batteries is of importance as it gives the basis for a battery management system which will be able to adapt to different batteries.

\section{References}

Berndt, D., 2001. Valve-regulated lead-acid batteries. Journal of Power Sources, Band 100, pp. 29-46.

Buller, S., Thele, M., Karden, E., Doncker, R. D., 2003. Impedance-based non-linear dynamic battery modeling for automotive applications. Journal of Power Sources, Band 113, pp. 422-430.

Bullock, K. and Salkind, A., 2011. Valve-regulated lead-acid batteries Chapter 17. In: Linden's Handbook of Batteries Fourth Edition. s.1.:s.n.

Cugnet, M. et al., March 2010. On Lead-Acid-Battery Resistance and CrankingCapability Estimation. IEEE Transactions on Industrial Electronics, 3(57), pp. 909917.

Dost, P. and Sourkounis, C., Nov. 2012. General Realisation Issues of a Battery Management System. In: Cyprus: 1st Conference on Power Options for the Eastern Mediterranean Region (POEM), pp. 147-152.

Eifert, M., 8-10 Oct. 2014. A discrete battery state monitoring algorithm for lead-acid batteries. 2014 IEEE Conference on Control Applications (CCA), pp. 1119-1126.

$\mathrm{Gu}$, H., 1990. Applications of mathematical models in lead-acid battery design. Aerospace and Electronic Systems Magazine, IEEE, 5(8), pp. 3-6.

Iwata, I. K. a. M., 19-23 Oct. 1997. Internal resistance and deterioration of VRLA battery - analysis of internal resistance obtained by direct current measurement and its application to VRLA battery monitoring technique. In: s.l.:Telecommunications Energy Conference 97, pp. 687-694.

Kerley, R., Hyun, J. H., Ha, D. S., Nov. 2015. Automotive lead-acid battery state-ofhealth monitoring system. Industrial Electronics Societ - IECON 2015 - 41st Annual Conference of the IEEE, pp. 003934-003938. 
Lambert, D., Greenwood, P., Reed, M., 2002. Advances in gelled-electrolyte technology for valve-regulated lead-acid batteries. Journal of Power Sources, 2(107), pp. 173179.

Mariani, A. et al., 11-12 Sept. 2015. Techniques for monitoring and predicting the OCV for VRLA battery systems. 2015 21st International Conference on Automation and Computing (ICAC), pp. 1-6.

Mauracher, P. and Karden, E., 1-4 Oct. 1996. Dynamic modeling of lead-acid batteries using impedance spectroscopy for parameter identification, Barcelona, Spain: 56th European Lead Battery Conf. (5ELBC).

Piller, S., Perrin, M., Jossen, A., 2001. Methods for state-of-charge determination and their applications. Journal of Power Sources, Band 96, pp. 113-120.

Salameh, Z., Casacca, M., Lynch, W., 1992. A mathematical model for lead-acid batteries. Energy Conversion, IEEE, 7(1), pp. 93-98.

Salkind, A. and Zguris, G., 2011. Lead-acid batteries Chapter 16. In: Linden's Handbook of Batteries Fourth Edition. s.l.:s.n.

Salomon, M., 2011. Electrochemical principles and reactions. In: Linden's Handbook of Batteries Fourth Edition, Chapter 2. s.1.:s.n.

Ushiyama, K. and Morimoto, M., 5-8 Dec. 2011. SOH Estimation of Lead Acid Battery for Automobile. In: s.1.:Power Electronics and Drive Systems (PEDS), pp. 739-744.

Zhang, J. et al., 2012. Electrochemical technologies for energy storage and conversion. Wiley, pp. 143-169. 
